\title{
In vitro activity of fosfomycin in combination with linezolid against clinical isolates of methicillin-resistant Staphylococcus aureus
}

\begin{abstract}
Yu Xu-hong ${ }^{1}$, Matthew E Falagas ${ }^{2,3,4}$, Wang Dong ${ }^{5}$, Drosos E Karageorgopoulos ${ }^{2}$, Lin De-feng ${ }^{6}$ and Wang Rui $^{7}$
The objective of this paper was to investigate the in vitro effects of fosfomycin combined with linezolid against methicillin-resistant Staphylococcus aureus (MRSA). A total of 102 MRSA isolates isolated from clinical specimens of human infections from three hospitals in China were studied. The microdilution checkerboard method was used to determine whether combinations act synergistically against these isolates. The susceptibility results for fosfomycin and linezolid were interpreted according to the guidelines of the Clinical and Laboratory Standards Institute. Synergy and indifference were defined as a fractional inhibitory concentration index of $\leqslant 0.5$ and $>0.5$ but $\leqslant 4$, respectively. The combination of fosfomycin and linezolid demonstrated the following interactions: $98.04 \%$ (100/102) synergism; $1.96 \%(2 / 102)$ indifference; no antagonism was seen. Thus, the combination between fosfomycin and linezolid shows synergism for most of the MRSA isolates tested in this study. If these findings are confirmed in further in vitro or in vivo studies, the above combination could be tested clinically for difficulty to treat MRSA infections, particularly those warranting prolonged oral therapy.
\end{abstract}

The Journal of Antibiotics (2014) 67, 369-371; doi:10.1038/ja.2014.5; published online 12 February 2014

Keywords: drug synergism; fosfomycin; fractional inhibitory concentration index; linezolid; methicillin-resistant Staphylococcus aureus; MRSA

\section{INTRODUCTION}

Methicillin-resistant Staphylococcus aureus (MRSA) can cause serious or even life-threatening infections in humans, such as pneumonia, bacteremia and endocarditis, often with septic pulmonary emboli, bone and joint infections, or extensive soft tissue infections. These infections are associated with considerable attributable mortality and morbidity as well as health-care costs. ${ }^{1,2}$ The prevalence of MRSA infections has increased worldwide. ${ }^{3,4}$ Currently, over $60 \%$ of the S. aureus isolates from intensive care units (ICUs) in the United States of America and over 50\% of such isolates from inpatient non-ICU hospital settings are MRSA. ${ }^{5,6}$ More than $60 \%$ of the nosocomial S. aureus isolates in China are also MRSA. ${ }^{7}$ In Hong Kong hospitals, MRSA has been endemic since the mid 1980s. ${ }^{8}$

Vancomycin is an effective agent in treating serious infections due to MRSA. ${ }^{9}$ However, other treatment options should be explored for patients that cannot tolerate vancomycin or, perhaps, in the case of infection with MRSA strains that show heteroresistance or intermediate susceptibility to this drug. ${ }^{10}$ In recent years, new antistaphylococcal antibiotics, such as linezolid, daptomycin, tigecycline or telavancin have been developed. A number of older antimicrobial agents, such as fosfomycin and fusidic acid, are reemerging as potentially valuable additions in the armamentarium against staphylococcal infections. ${ }^{11}$

Linezolid is the first available synthetic antibiotic of the oxazolidinone class, which blocks protein synthesis at the ribosome and prevents the formation of the initiation complex. ${ }^{12,13}$ Linezolid is active in vitro against Gram-positive bacteria, including multiresistant strains. ${ }^{14-17}$ Still, linezolid-resistant MRSA was first reported in $2001 .^{18}$ Linezolid, which has been approved for the treatment of staphylococcal skin and soft-tissue infections or pneumonia, has been found to be clinically useful for the treatment of various other infections. ${ }^{19-21}$ An important feature of linezolid is its high oral bioavailability and excellent tissue penetration. ${ }^{22}$ Yet, linezolid has been associated with a greater risk of thrombocytopenia compared with vancomycin. ${ }^{23}$

Fosfomycin is a phosphoenolpyruvate analog that binds to UDP- $N$-acetylglucosamine enolpyruvyl transferase (MurA) inhibiting the formation of $\mathrm{N}$-acetyl-muramic acid, the first committed step in bacterial cell wall synthesis. ${ }^{24,25}$ Fosfomycin is bactericidal against MRSA and has also been reported to directly interact with the immune system by affecting T-lymphocyte function. ${ }^{26-28}$

${ }^{1}$ Department of Pharmacy, The Chinese PLA 305 Hospital, Beijing, China; ${ }^{2}$ Alfa Institute of Biomedical Sciences (AlBS), Athens, Greece; ${ }^{3}$ Department of Medicine, Henry Dunant Hospital, Athens, Greece; ${ }^{4}$ Department of Medicine, Tufts University School of Medicine, Boston, MA, USA; ${ }^{5}$ The Chinese PLA General Hospital, Pharmaceutical Administration, Beijing, China; ${ }^{6}$ Department of Pharmacy, Skin Disease Prevention and Control Hospital, Qanzhou, Fujian, China and ${ }^{7}$ Department of Clinical Pharmacology, The Chinese PLA General Hospital, Beijing, China

Correspondence: Professor W Rui, Department of Clinical Pharmacology, the PLA General Hospital, Beijing 100853, China. E-mail: ruiwang301@sohu.com

Received 22 November 2011; revised 2 November 2012; accepted 14 January 2014; published online 12 February 2014 
Table 1 Minimum inhibitory concentration (MIC) (in $\mathrm{mg} \mathrm{I}^{-1}$ ) of fosfomycin combined with linezolid in MRSA $(N=102)$

\begin{tabular}{|c|c|c|c|c|c|c|}
\hline \multirow[b]{2}{*}{ Antimicrobial } & \multicolumn{3}{|c|}{ Single } & \multicolumn{3}{|c|}{ Combination } \\
\hline & $M I C_{50}$ & $M I C_{90}$ & MIC range & $M I C_{50}$ & $M I C_{90}$ & MIC range \\
\hline Fosfomycin & 64 & 128 & $16 \sim 128$ & 8 & 16 & $2 \sim 32$ \\
\hline Linezolid & 1 & 2 & $0.25 \sim 4$ & 0.25 & 0.5 & $0.125 \sim 1$ \\
\hline
\end{tabular}

The objective of this study was to evaluate the in vitro effects of the combination between fosfomycin and linezolid against MRSA isolates.

\section{MATERIALS AND METHODS}

\section{Bacterial isolates}

We collected and evaluated 102 clinical MRSA isolates from three Chinese hospitals (specifically, 56 isolates from PLA General Hospital, 21 isolates from Beijing Hospital and 25 isolates from Peking Union Medical College Hospital). The sites of isolation were blood (39\%), sputum (24\%), nose (11\%), wounds $(19 \%)$ or other unspecified site $(7 \%)$. Species identification was done using the automated VITEK-2 system (bioMérieux, Marcy l'Etoile, France) or the SLIDEX Staph Plus (bioMérieux), a rapid latex agglutination test. S. aureus ATCC 25923 was used as the quality control strain in the latex agglutination test. The agar disk diffusion (Kirby-Bauer) method was used for routine antimicrobial susceptibility testing. Methicillin resistance was detected using a $30-\mu \mathrm{g}$ cefoxitin disk.

\section{Study antimicrobials}

Fosfomycin was obtained from the National Institute for the Control of Pharmaceutical and Biological Products (Beijing, China). The purity of fosfomycin was 99\%. Linezolid was obtained from Pfizer Pharmaceuticals Limited. Antibiotic powders were used to prepare stock solutions at a concentration of $1024 \mathrm{mgl}^{-1}$.

\section{MIC determination and synergy testing}

Initially, all study isolates were tested against the two study antimicrobials and minimum inhibitory concentrations (MICs) were determined using the broth microdilution method, according to the Clinical and Laboratory Standards Institute (CLSI) standards. ${ }^{29}$ S. aureus ATCC 29213 was used as a quality control strain. ${ }^{29}$

The checkerboard broth microdilution method was used for synergy testing between fosfomycin and linezolid. Synergy testing was performed in 96-well broth microdilution trays containing the two study antimicrobial agents in twofold dilutions dispensed in a checkerboard format. ${ }^{30}$ Fosfomycin was dispensed in the first row with concentration ranging from $1 \mathrm{mgl}^{-1}$ to $512 \mathrm{mgl}^{-1}$, whereas linezolid was dispensed in the first column with concentration ranging from $0.25 \mathrm{mgl}^{-1}$ to $16 \mathrm{mgl}^{-1}$. These concentrations were set according to the MIC values obtained in the preliminary susceptibility tests.

Bacterial inocula were prepared by suspending growth from agar plates in Mueller-Hinton broth to a density of $0.5 \mathrm{McF}$ arland standard and were diluted 1:10 to produce a final inoculum of $1.5 \times 10^{5}$ colony-forming units per $\mathrm{ml}$ with a multipoint inoculator. The trays were incubated aerobically overnight.

The interpretation of the effect of the combination of the two study antimicrobials was based on the calculation of the fractional inhibitory concentration index (FICI). The FICI was calculated according to the following formula: ${ }^{31}$

$$
\begin{aligned}
\mathrm{FICI}= & (\mathrm{FIC} \text { of DrugA })+(\text { FIC of DrugB }) \\
= & (\text { MIC of DrugA in combination } / \text { MIC of DrugA alone }) \\
& +(\text { MIC of DrugB in combination/MIC of DrugB alone }) .
\end{aligned}
$$

Antagonism was reported if the maximum FICI was $>4$; synergy if the minimum FICI was $\leqslant 0.5$; and indifference was reported for all the intermediate results $(0.5<\mathrm{FICI} \leqslant 4) .{ }^{32}$

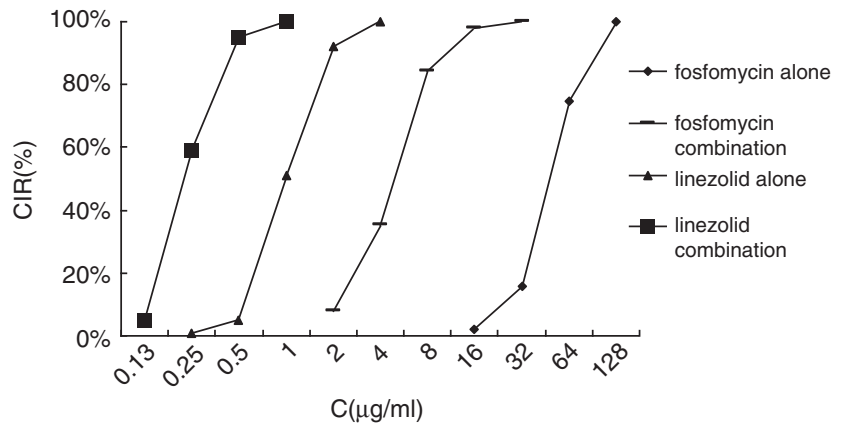

Figure 1 Cumulative inhibition ratio (CIR) of fosfomycin and/or linezolid against MRSA ( $N=102)$. C, concentration.

The concentration of each of the two study antimicrobials in the combination that corresponded to the minimum FICI observed for each tested isolate was recorded. This concentration was used to calculate the $\mathrm{MIC}_{50}$ and $\mathrm{MIC}_{90}$ of each antibiotic in combination with the other.

\section{RESULTS}

For the 102 MRSA isolates studied, the $\mathrm{MIC}_{50}$ and $\mathrm{MIC}_{90}$ of fosfomycin alone were $64 \mathrm{mgl}^{-1}$ and $128 \mathrm{mgl}^{-1}$, respectively, and those of linezolid alone were $1 \mathrm{mgl}^{-1}$ and $2 \mathrm{mgl}^{-1}$, respectively. The $\mathrm{MIC}_{50}$ and $\mathrm{MIC}_{90}$ of fosfomycin in combination with linezolid were $8 \mathrm{mgl}^{-1}$ and $16 \mathrm{mgl}^{-1}$, respectively, and those of linezolid in combination with fosfomycin were $0.25 \mathrm{mgl}^{-1}$ and $0.5 \mathrm{mgl}^{-1}$, respectively (Table 1 ). The cumulative inhibition ratio (CIR) for fosfomycin and linezolid against MRSA, both alone and in combination, is shown in Figure 1. The cumulative inhibition curves for both antibiotics shifted to the left when they were used together, compared with when used alone.

The combination of fosfomycin and linezolid showed synergy against $100(98.04 \%)$ of the 102 MRSA isolates studied and indifference against the remaining 2 isolates $(1.96 \%)$. No antagonism was observed. For $89(87.3 \%)$ of the 102 isolates, the minimum FICIs were obtained with a linezolid FIC of 0.25 (that is, with a linezolid concentration in the combination of $\frac{1}{4}$ times the MIC of linezolid alone). Overall, the most frequent combinations of the two antibiotics, with which the minimum FICIs were obtained, were fosfomycin FIC of $0.125 \times$ linezolid FIC of 0.25 ( 39 isolates), fosfomycin FIC of $0.0625 \times$ linezolid FIC of 0.25 (28 isolates), fosfomycin FIC of $0.25 \times$ linezolid FIC of 0.25 (15 isolates), fosfomycin FIC of $0.03125 \times$ linezolid FIC of 0.25 ( 6 isolates).

\section{DISCUSSION}

In this study, fosfomycin combined with linezolid was synergistic in vitro against almost all (98\%) of 102 clinical MRSA isolates collected in hospitals in China. No antagonism between the two drugs was observed. Most of the isolates had MIC values within the susceptible range for linezolid, whereas only $74.5 \%$ did so for fosfomycin. The CIR curves show a notable difference toward increased susceptibility for both fosfomycin and linezolid in combination.

The above in vitro findings suggest that a fosfomycin-linezolid combination regimen for the treatment of serious MRSA infections could be clinically useful. This is important as linezolid has a bacteriostatic mode of action, which raises concerns for the utility of this agent for the treatment of infectious syndromes for which bactericidal activity is desirable, such as bacteremia or endocarditis. ${ }^{33,34}$ In vivo studies have shown that the combination of 
fosfomycin with linezolid against experimental staphylococcal infections can result in increased efficacy. ${ }^{31,35}$

Combination therapy with the fosfomycin-linezolid regimen may also be particularly important for the treatment of staphylococcal infections by the oral route. If this combination proves to have increased clinical efficacy compared with linezolid alone, this could potentially lead to treatment courses of shorter duration. Then, some of the adverse events associated with prolonged treatment with linezolid, such as myelosuppression and peripheral neuropathy, could be prevented. ${ }^{36}$ The use of a fosfomycin-linezolid combination regimen could also prevent the emergence of resistance during therapy. This is a particular concern with fosfomycin therapy, due to a high mutation rate to fosfomycin resistance observed in vitro. ${ }^{37,38}$

MRSA has re-emerged as a global public health problem. ${ }^{39}$ Over the last decade, MRSA has become increasingly common in hospitals and in community settings. ${ }^{40,41}$ Combination antimicrobial therapy is an important treatment approach for infections. Synergy is one of the most common reasons for using combination antimicrobial therapy. Thus, the search for combinations of antibiotics might yield more effective treatment options and fewer side effects. Some compounds are unable to inhibit or kill bacteria by themselves, but can block bacterial mechanisms of resistance, enhancing the activity of other antimicrobials administered in combination.

In conclusion, the combination of fosfomycin with linezolid showed synergistic activity in vitro against almost all of the isolates of MRSA that were studied. The use of a combination regimen with fosfomycin and linezolid could theoretically enhance treatment efficacy, prevent the emergence of resistance or even decrease toxicity by limiting the duration of therapy compared with linezolid alone. Further studies, especially in animal models of infection, are required to establish whether the in vitro synergistic activity observed with fosfomycin and linezolid in our study can be translated to higher therapeutic efficacy in vivo.

\section{ACKNOWLEDGEMENTS}

The project was supported by the National Natural Science Foundation of China (No. 30873127).

1 Lodise, Jr T. P. \& McKinnon, P. S. Burden of methicillin-resistant Staphylococcus aureus: focus on clinical and economic outcomes. Pharmacotherapy 27, 1001-1012 (2007).

2 Shorr, A. F. et al. Morbidity and cost burden of methicillin-resistant Staphylococcus aureus in early onset ventilator-associated pneumonia. Crit. Care 10, R97 (2006).

3 Thornsberry, C. Epidemiology of staphylococcal infections-a USA perspective. J. Chemother. 6, 61-65 (1994).

4 Vandenbroucke-Grauls, C. Epidemiology of staphylococcal infections-a European perspective. J. Chemother. 6, 67-70 (1994).

5 Centers for Disease Control and Prevention (CDC). MRSA among ICU Patients (1995-2004).

6 National Nosocomial Infections Surveillance System. National Nosocomial Infections Surveillance (NNIS) System Report, data summary from January 1992 through June 2004, issued October 2004. Am. J. Infect. Control 32, 470-485 (2004).

7 Wang, F., Zhu, D. M., Hu, F. P. \& Zhang, Y. Y. Surveillance of bacterial resistance among isolates in Shanghai in 1999. J. Infect. Chemother. 7, 117-120 (2001).

8 Ho, P., Yuen, K., Yam, W., Sai-yin Wong, S. \& Luk, W. Changing patterns of susceptibilities of blood, urinary and respiratory pathogens in Hong Kong. J. Hosp. Infect. 31, 305-317 (1995).

9 Craven, D. E., Kollisch, N. R., Hsieh, C. R., Connolly, Jr M. G. \& McCabe, W. R. Vancomycin treatment of bacteremia caused by oxacillin-resistant Staphylococcus aureus: comparison with beta-lactam antibiotic treatment of bacteremia caused by oxacillin-sensitive Staphylococcus aureus. J. Infect. Dis. 147, 137-143 (1983).

10 Sorrell, T. C., Packham, D. R., Shanker, S., Foldes, M. \& Munro, R. Vancomycin therapy for methicillin-resistant Staphylococcus aureus. Ann. Intern. Med. 97, 344-350 (1982).

11 Falagas, M. E., Grammatikos, A. P. \& Michalopoulos, A. Potential of old-generation antibiotics to address current need for new antibiotics. Expert Rev. Anti Infect. Ther. 6 , 593-600 (2008).
12 Shinabarger, D. L. et al. Mechanism of action of oxazolidinones: effects of linezolid and eperezolid on translation reactions. Antimicrob. Agents Chemother. 41, 2132-2136 (1997).

13 Zurenko, G. E. et al. In vitro activities of U-100592 and U-100766, novel oxazolidinone antibacterial agents. Antimicrob. Agents Chemother. 40, 839-845 (1996).

14 Clemett, D. \& Markham., A. Linezolid. Drugs 59, 815-827, discussion 828 (2000). 15 Diekema, D. I. \& Jones., R. N. Oxazolidinones: a review. Drugs 59, 7-16 (2000).

16 Gemmell, C. G. Susceptibility of a variety of clinical isolates to linezolid: a European inter-country comparison. J. Antimicrob. Chemother. 48, 47-52 (2001).

17 Swaney, S. M., Aoki, H., Ganoza, M. C. \& Shinabarger, D. L. The oxazolidinone linezolid inhibits initiation of protein synthesis in bacteria. Antimicrob. Agents Chemother. 42, 3251-3255 (1998).

18 Tsiodras, S. et al. Linezolid resistance in a clinical isolate of Staphylococcus aureus. Lancet 358, 207-208 (2001).

19 Chien, J. W., Kucia, M. L. \& Salata, R. A. Use of linezolid, an oxazolidinone, in the treatment of multidrug-resistant gram-positive bacterial infections. Clin. Infect. Dis. 30, 146-151 (2000)

20 Jones, R. N., Ballow, C. H. \& Biedenbach, D. J. Multi-laboratory assessment of the linezolid spectrum of activity using the Kirby-Bauer disk diffusion method: Report of the Zyvox Antimicrobial Potency Study (ZAPS) in the United States. Diagn. Microbiol. Infect. Dis. 40, 59-66 (2001).

21 Perry, C. M. \& Jarvis., B. Linezolid: a review of its use in the management of serious gram-positive infections. Drugs 61, 525-551 (2001).

$22 \mathrm{Gee}, \mathrm{T}$. et al. Pharmacokinetics and tissue penetration of linezolid following multiple oral doses. Antimicrob. Agents Chemother. 45, 1843-1846 (2001).

23 Beibei, L. et al. Linezolid versus vancomycin for the treatment of gram-positive bacterial infections: meta-analysis of randomised controlled trials. Int. J. Antimicrob. Agents 35, 3-12 (2010).

24 Forsgren, A. \& Walder, M. Antimicrobial activity of fosfomycin in vitro. J. Antimicrob. Chemother. 11, 467-471 (1983).

25 Grif, K., Dierich, M. P., Pfaller, K., Miglioli, P. A. \& Allerberger, F. In vitro activity of fosfomycin in combination with various antistaphylococcal substances. J. Antimicrob. Chemother. 48, 209-217 (2001).

26 Falagas, M. E. et al. Antimicrobial susceptibility of Gram-positive non-urinary isolates to fosfomycin. Int. J. Antimicrob. Agents 35, 497-499 (2010).

27 Falagas, M. E., Roussos, N., Gkegkes, I. D., Rafailidis, P. I. \& Karageorgopoulos, D. E. Fosfomycin for the treatment of infections caused by Gram-positive cocci with advanced antimicrobial drug resistance: a review of microbiological, animal and clinical studies. Expert Opin. Investig. Drugs 18, 921-944 (2009).

28 Morikawa, K., Oseko, F., Morikawa, S. \& Sawada, M. Immunosuppressive activity of fosfomycin on human T-lymphocyte function in vitro. Antimicrob. Agents Chemother. 37, 2684-2687 (1993).

29 Clinical and Laboratory Standards Institute. Performance standards for antimicrobial susceptibility testing; Nineteenth Informational Supplement. CLSI document M100-S19 (CLSI, Wayne, PA, USA, 2009).

30 Moody, J. Synergism testing: broth microdilution checkerboard and broth macrodilution method. In C. m.-b. p. handbook (ed. Isenberg, HD) vol 2nd edn p 1-28 (ASM Press, Washington, DC, USA, 2004).

31 Sahuquillo Arce, J. M. et al. In vitro activity of linezolid in combination with doxycycline, fosfomycin, levofloxacin, rifampicin and vancomycin against methicillin-susceptible Staphylococcus aureus. Rev. Esp. Quimioter. 19, 252-257 (2006).

32 Odds, F. C. Synergy, antagonism, and what the chequerboard puts between them. J. Antimicrob. Chemother. 52, 1 (2003).

33 Falagas, M. E., Manta, K. G., Ntziora, F. \& Vardakas, K. Z. Linezolid for the treatment of patients with endocarditis: a systematic review of the published evidence. J. Antimicrob. Chemother. 58, 273-280 (2006).

34 Jang, H. C. et al. Salvage treatment for persistent methicillin-resistant Staphylococcus aureus bacteremia: efficacy of linezolid with or without carbapenem. Clin. Infect. Dis. 49, 395-401 (2009).

35 Pachon-Ibanez, M. E. et al. Efficacy of fosfomycin and its combination with linezolid, vancomycin and imipenem in an experimental peritonitis model caused by a Staphylococcus aureus strain with reduced susceptibility to vancomycin. Eur. J. Clin. Microbiol. Infect. Dis. 30, 89-95 (2011).

36 Bressler, A. M., Zimmer, S. M., Gilmore, J. L. \& Somani, J. Peripheral neuropathy associated with prolonged use of linezolid. Lancet. Infect. Dis. 4, 528-531 (2004).

37 Courtieu, A. L., Drugeon, H. \& Billaudel, S. Susceptibility to fosfomycin of hospital strains isolated in Nantes (France). Frequency of mutation to resistance. Chemotherapy 23, 25-36 (1977).

38 Roussos, N., Karageorgopoulos, D. E., Samonis, G. \& Falagas, M. E. Clinical significance of the pharmacokinetic and pharmacodynamic characteristics of fosfomycin for the treatment of patients with systemic infections. Int. J. Antimicrob. Agents 34, 506-515 (2009).

39 Grundmann, H., Aires-de-Sousa, M., Boyce, J. \& Tiemersma, E. Emergence and resurgence of meticillin-resistant Staphylococcus aureus as a public-health threat. Lancet 368, 874-885 (2006).

40 Moreno, F., Crisp, C., Jorgensen, J. H. \& Patterson., J. E. Methicillin-resistant Staphylococcus aureus as a community organism. Clin. Infect. Dis. 21, 1308-1312 (1995).

41 Robinson, D. A. et al. Re-emergence of early pandemic Staphylococcus aureus as a community-acquired meticillin-resistant clone. Lancet 365, 1256-1258 (2005). 